\title{
ON THE FORMATION OF PREFERRED INCLUSION SHAPE ORIENTATIONS IN COPPER-BASED COMPOSITES
}

\author{
A. N. NIKITIN ${ }^{1}$, T. I. IVANKINA ${ }^{2}$ and I. K. ARKHIPOV \\ ${ }^{1)}$ Joint Institute for Nuclear Research, Dubna, Russia \\ ${ }^{2)}$ Teacher's Training University, Tula, Russia \\ ${ }^{3)}$ Tula Technical University, Tula, Russia
}

(Received 19 December 1995)

\begin{abstract}
A three-component model of copper-based composite loaded by tensile forces is proposed. The effect of a nonhydrostatic stress state and the formation of microplastic zones cause the transition of the composite from an isotropic to an anisotropic state. We calculate the tensor of effective elastoplastic modulii of the model as a function of the applied load and of the microplastic zone concentration.
\end{abstract}

KEY WORDS: "Shape texture", microplastic zone, effective elastoplastic moduli.

\section{INTRODUCTION}

In polycrystalline materials (metals, alloys, rocks), as well as in grain composites, anisotropy is conditioned mainly by preferred orientations of crystallites or of inclusions of similar shapes. In the case of crystallites, the material is said to have a texture and in the case of inclusions their shape can vary with applied load etc. In the future text we call the variation of the shape of the inclusion "shape texture".

Many publications, e.g. (Wenk, 1985; Wenk, 1994; Siemes and Henning-Michaeli, 1985; Jonas et al., 1994; Nikitin and Ivankina, 1995), were dedicated to the study of conditions and mechanisms of the texture formation. Considerably less attention was given to the investigation of processes of the development of "shape textures". This type of textures, however, is common in the construction materials, geomaterials, and grain composites which have experienced plastic deformation.

Of interest are such models of texture formation which permit the calculation of the type of induced anisotropy (symmetry of material effective tensors) in dependence on the type of applied force (symmetry of the field tensors of deformation and stress) and the characteristics of the medium itself.

The present paper suggests a model for the development of shape texture and, as a consequence, of the anisotropy of elastic properties in a copper-based composite. The technology of manufacturing such composites results in a chaotic distribution of rigid inclusions in a plastic matrix.

Thus, a composite in the initial state (without any external loads) is an isotropic microinhomogenous body, whose mechanical characteristics are described by statistically isotropic and statistically homogeneous random fields. 
As a result of external stress, a third component arises: in the vicinity of the matrixinclusion interface there are zones of microplasticity. Their forms and sizes depend on the type and magnitude of the macroscopic stress. The other two components of the model in an undistorted state are iron crystallites (rigid inclusions) and isotropic polycrystalline associations of copper crystallites (soft matrix).

It is the main property of the third component that the yield stress $\tau_{s}$, is reached earlier than in the other components of the model. As a consequence, under the action of external mechanical stress less $\tau_{s}$, both the matrix and inclusions are deformed elastically, and the microplasticity zone undergoes plastic deformation. In this case, the deformation compatibility principle holds along the entire inclusion and matrix boundary.

Let us discuss in more detail the considerations that allow the introduction of a third component into the model.

In an other model proposed in (Nikitin and Arkhipov, 1992), there was considered a quartz-bearing polycrystalline rock which was deformed at a temperature corresponding to the $\alpha-\beta$ phase transition, and the microplasticity zone arose due to the anomalous properties of quart. The $\alpha$-quartz layer which surrounds the inclusion has a negative Poisson coefficient over the temperature range from $573^{\circ} \mathrm{C}$ to $524^{\circ} \mathrm{C}$ and, as a consequence, the transverse dimensions of the material increase on stretching. This special feature of quartz leads to large local strains at the phase interface, i.e., in the area defined as a zone of microplasticity.

In the case of grain composites or powder metallurgy products, the zone of microplasticity can be defined as an area of concentration of microstresses. These microstresses can appear in the process of manufacturing or operating industrial products or their parts, and the character of their distribution and values influences the effective physical and mechanical characteristics of the material. In the microplastic zones the mechanical characteristics have local values, and one can therefore expect that in the vicinity of the microzone the local stress values will earlier reach the yield stress value with increasing external stress.

A special feature of internal stresses in an inhomogeneous medium are the fluctuations and concentrations of fields at the component interface and in their vicinity. To investigate the distribution and values of internal stresses a method of local strain measurements, as well as other methods of layer-by-layer analysis (Kasatkin et al., 1981; Fink and Rorbach, 1961; Webster et al., 1988) are applied. But neutron diffraction only permits determining the distribution of microstresses over the bulk volume of industrial product without destroying them (Allen et al., 1985). According to neutron diffraction data, the residual microstress values (e.g., in an aluminum weld) vary from 0 to 140 $\mathrm{MPa}$.

\section{A MODEL FOR A COMPOSITE WITH MICROPLASTIC ZONES}

Let us describe the model under stress. Under the action of external stretching stresses which are not equal and slowly increase, microplastic zones arise after the stresses have achieved a certain value. We are considering the case of "simple active stresses" when the stresses increase proportional to a phenomenic parameter (Ilyushin, 1963).

Next we assume that for a certain intensity level of applied stress, the microplastic zone completely occupies the inclusion, and both the zone and inclusion together take the shape of an ellipsoid (see Figure 1). Such a structure is replaced by a system of so called effective grains, ellipsoids, which consist of an elastic kernel (inclusion) and 


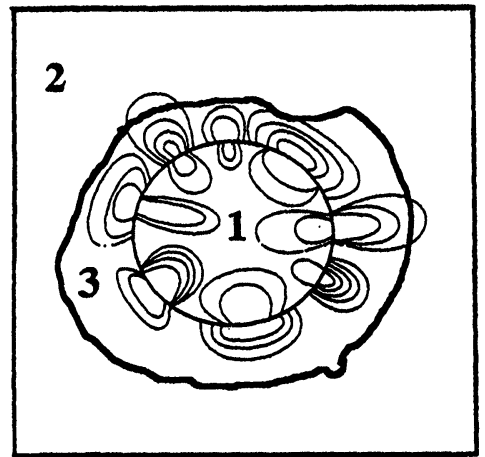

(a)

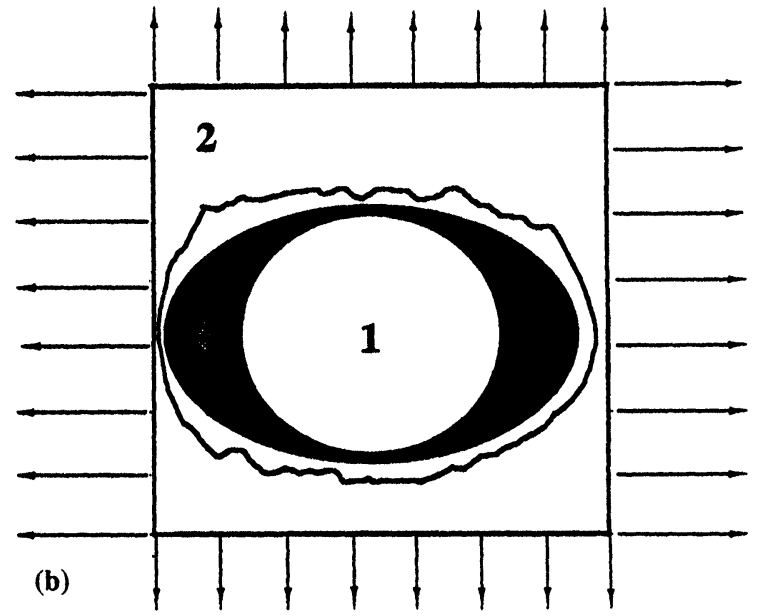

Figure 1a The model of an iron-copper powder composite in an undistorted state: component 1 - rigid inclusion; component 2 -plastic matrix; component 3 -area of microstress concentrations.

Figure 1b The same model under the action of nonequilibrium external stresses: component 1 -rigid inclusion; component 2 -plastic matrix; component 3 -microplastic zone.

an external plastic part. These grains are submerged in a matrix with effective properties. The concentration of microplastic zones and dimensions of ellipsoids change when the applied stresses increase.

The type of anisotropy depends on the type of external stress and variable characteristics of the inhomogeneous model, on the coefficient of shape anisotropy and on the concentration of microplastic zones. In order to calculate the tensor of effective modules you have to solve two interrelated problems:

1. Determination of the shape and dimensions of the effective grain from the type and amount of external stress;

2. Calculation of the effective elastoplastic characteristics of an inhomogeneous composite from the shape of effective grain.

\section{Solution of Problem 1}

In the following we consider the case of external symmetry of stresses $\sigma_{11}^{0}=\sigma_{22}^{0} \neq \sigma_{33}^{0}$. We define the anisotropy coefficient $k$ of the effective microplasticity zone as

$$
k^{2}=1-\frac{a^{2}}{c^{2}}
$$

where $a$ and $c$ are the lengths of semiaxes of the ellipsoid enclosing the elastic inclusion, in the direction of the reference system axes $x_{1}$ and $x_{3}$ respectively. By the self-consistent method (Shermergor, 1977), the total strain $\sigma$ in a microplastic grain is determined as:

$$
\sigma=c^{*}\left(\varepsilon^{c}-\varepsilon^{T}+\varepsilon^{o}\right)
$$


where $c^{*}$ is the effective elastoplastic stiffness of the grain consisting of the inclusion and the plastic part of matrix, $\varepsilon^{c}$ is the restricted deformation in the grain, $\varepsilon^{T}$ is the residual deformation in the grain, $\varepsilon^{0}$ is the deformation caused by external homogeneous stresses. In order to calculate the intensity of stresses $\sigma_{i}$ from components of the tensor $\sigma$ and with account for the von Mises elasticity condition, $\sigma_{i}=\sigma_{s 2}$, where $\sigma_{s 2}$ is the matrix yield limit, instead of (2) holds:

$$
v_{1} \sigma_{i 1}+v_{2} \sigma_{s 2}=2 \mu^{*}\left(\varepsilon_{i}^{c}-\varepsilon_{i}^{T}+\varepsilon_{i}^{0}\right)
$$

Here $v_{1}$ and $v_{2}$ are the volume concentrations of the inclusion and the plastic part of matrix in the effective grain $\left(v_{1}+v_{2}=1\right), \sigma_{i 1}$ is the strain inside of the inclusion, $\mu^{*}$ is the effective elastoplastic shear, $\varepsilon_{i}^{c}, \varepsilon_{i}^{T}, \varepsilon_{i}^{0}$ are the corresponding values for the deformation.

As is known, for an ellipsoid inclusion the tensor components of restricted deformation and residual deformation are connected via the Rauss tensor N (Shermergor, 1977) as

$$
\varepsilon^{c}=\mathrm{N} \varepsilon^{T}
$$

Calculating the corresponding contraction tensor we get for the deformation

$$
\varepsilon_{t}^{c}=\bar{N} \varepsilon^{T}
$$

Taking into account that $\sigma_{i}^{0}=2 \mu_{i}^{*} \varepsilon_{i}^{0}$, the external stress intensities, instead of (3) we get

$$
v_{1} \sigma_{i 1}+v_{2} \sigma_{s 2}=2 \mu^{*}\left(\bar{N} \varepsilon^{T}-\varepsilon_{i}^{T}\right)+\sigma_{i}^{0}
$$

we find the residual deformation $\varepsilon^{T}$ from its definition

$$
\varepsilon^{T}=\varepsilon-\varepsilon_{d i s}
$$

where $\varepsilon_{\text {dis }}$ is the discharging deformation which, in the given case, is expressed through the Ilyushin's function as follows

$$
\varepsilon_{d i s}=\left(1-\omega\left(<e_{i}>\right)\right) \varepsilon
$$

where symbols standing in brackets " $<>$ " are mean values.

Substituting (7) into (6) we get

$$
\varepsilon^{T}=\omega\left(<e_{i}>\right) \varepsilon
$$

Taking into account (8), we rewrite (5) through tensor components

$$
\omega \sqrt{2}\left[\left(N_{11}-N_{31}+N_{12}-N_{32}\right)<\sigma_{11}>+\left(N_{13}-N_{33}\right)<\sigma_{33}>\right]=v_{1} \sigma_{i 1}+v_{2} \sigma_{s 2}-\sigma_{i}^{0}
$$

Here $N_{i j}$ are the Rauss tensor components for the ellipsoid inclusion. These components depend on the ratio of semiaxes $a / c$, or on the shape anisotropy parameter $k$. The Ilyushin's function $\omega$ is connected to the parameter $\chi$ by the relation

$$
\omega=1-\frac{\sigma_{s 2}}{\sigma_{i 1}}=1-\chi
$$


The parameter $\chi$ depends on the average deformation of the component $\left\langle e_{i}\right\rangle$, and can be expressed as

$$
\chi=\frac{\mu_{2}}{\mu_{1} \mathrm{~B}} \frac{e_{s 2}}{\left.<e_{i}\right\rangle}
$$

Substituting the Rauss tensor components and performing some algebraic transformations leads to the following equation for calculating the anisotropy parameter $k$

$$
\begin{gathered}
0.9(1-7 \eta) \rho^{4}-\left(1+0.5(1-\eta)-\frac{(4+\eta)(1-2 v)}{6}\right) \rho^{2}-\left(1-\frac{5(1-2 v)}{6}\right) \eta= \\
=\frac{2(1-v)}{\omega}(1-\eta)\left[\frac{\mu_{1} B}{\mu^{*}}\left(v_{1}+v_{2} \frac{\sigma_{s 2}}{\sigma_{i 1}}\right)-1\right]
\end{gathered}
$$

Here $\rho^{2}=1-k^{2}, \rho^{2}=\frac{a^{2}}{c^{2}}, \mathrm{~h}=\frac{\sigma_{33}^{0}}{\sigma_{11}^{0}}, v$ is the Poisson coefficient of the copper matrix, $\mu_{1}$ is the inclusion shear module, $\mu_{2}$ is the matrix shear module;

$$
\mathrm{B}=\frac{1-4 \beta_{2} \mathrm{~b}}{1-4 \beta \mathrm{b}}
$$

where $\beta=\left(\mathrm{v}_{1}-\mathrm{v}_{2}\right)\left(\mu_{1}-\mu_{2}\right)$

$$
\begin{aligned}
& \beta_{2}=-v_{1}\left(\mu_{1}-\mu_{2}\right) \\
& b=-\frac{<3 \lambda+8 \mu>}{30<\mu><1+2 \mu>} \\
& <3 \lambda+\mu>=v_{1}\left(3 \lambda_{1}+8 \mu_{1}\right)+v_{2}\left(3 \lambda_{2}+8 \mu_{2}\right) \\
& <\mu>=v_{1} \mu_{1}+v_{2} \mu_{2} \\
& <\lambda+2 \mu>=v_{1}\left(\lambda_{1}+2 \mu_{1}\right)+v_{2}\left(\lambda_{2}+2 \mu_{2}\right)
\end{aligned}
$$

$\lambda_{1}, \mu_{1}$ and $\lambda_{2}, \mu_{2}$ are Lame parameters of inclusion and matrix correspondently. The solution of the biquadratic equation (10) with respect to the parameters $\eta, v_{1}, \chi$ for real roots are summarized in Table 1 for the given ratios of external stress $\eta=1.5$;

Table 1 The qualities $\eta, \chi$ and $\rho^{2}$ obtained from eq(10).

\begin{tabular}{cccc}
\hline$\eta$ & $\chi$ & \multicolumn{2}{c}{$\rho^{2}$} \\
\hline \multirow{4}{*}{1.5} & & $\mathrm{v}_{1}=0.1$ & $\mathrm{v}_{1}=0.5$ \\
& & $\mathrm{v}_{2}=0.9$ & $\mathrm{v}_{2}=0.5$ \\
& 0.5 & 0.44 & 0.41 \\
& 0.9 & 0.73 & 0.48 \\
2.0 & 1.0 & 0.81 & 0.51 \\
& 1.5 & - & - \\
& 0.9 & 0.60 & - \\
3.0 & 1.0 & 0.72 & - \\
& 1.5 & - & - \\
& 0.9 & 0.39 & - \\
& 1.0 & 0.63 & 0.82 \\
\hline
\end{tabular}


2; 3, and the parameter $\chi$ taking into account the external stress influence, which was chosen for the ratios $\frac{e_{s 2}}{\left\langle e_{i}\right\rangle}=0.5 ; 0.9 ; 1 ; 1.5$. In the calculations, the values of modules $\mu_{1}=0.8510^{5} \mathrm{MPa}, \mu_{2}=0.410^{5} \mathrm{MPa}, \lambda_{1}=1.27510^{5} \mathrm{MPa}$, and $\lambda_{2}=0.610^{5} \mathrm{MPa}$ were used.

\section{Solution of Problem 2.}

The effective elastoplastic stiffnesses of a composite is calculated from the shape of the effective grain. The elastoplastic shear of the plastic component is defined by the law of small elastoplastic deformations (Ilyushin, 1963):

$$
\mu_{*}^{(2)}=\mu_{2}\left(1-\omega\left(<e_{i}^{(2)}>\right)\right)
$$

$\mu_{2}$ - shear module of the matrix;

$\left.\omega\left(<e_{i}^{(2)}\right\rangle\right)$ - Ilyushin's function;

$\left.\left(<e_{i}^{(2)}\right\rangle\right)$ - deformation mean of the matrix;

To determine the effective elastoplastic stiffnesses of the three-component model the method of generalized singular approximation (GSA) (Shermergor, 1977) is applied. For the effective elastoplastic stiffnesses $c^{*}$ we use the following relationship:

$$
\begin{aligned}
& \left(c^{*}+b^{c}\right)^{-1}=\left\langle\left(c+b^{c}\right)^{-1}\right\rangle \\
& g\left(c^{c}+b^{c}\right)=-I
\end{aligned}
$$

where $I$ is the unit tensor, and the dependence of the tensor $g$ on the anisotropy parameter is given by the formula:

$$
\begin{aligned}
& g_{i j k l}=a_{i)(k, j)(l} \\
& a_{i j k l}=\frac{1}{8 \pi^{3}} \iint \bar{G}_{i j, k l} e^{-i \alpha r} d r d \alpha \\
& \bar{G}=\int G(\vec{r})^{i \alpha \vec{r}} d \vec{r}
\end{aligned}
$$

$G(\vec{r})$ is the Green tensor, $\vec{r}$ is the radius-vector of point. In the equation (15), the symmetrization operation is denoted by parentheses, so, for example, $a_{i)(k, j)(l} \equiv$ $\frac{1}{2}\left(a_{i(k, j) l}+a_{l(k, j) i}\right)$.

In (16) and (17), integrating is performed over the volume of the effective inhomogeneity area which is the ellipsoid. Following the integration in (16) and symmetrization in (15) we establish that the tensor $g_{i j k l}$ depends on the shape of the inhomogeneity surface and is expressed through the shape anisotropy coefficient $k$. After some transformations have been performed, the formulas (15) have the form

$$
g_{i j k l}=g_{i j k l}^{0}+k^{2} \phi_{i j k l}
$$

where $g_{i j k l}^{0}$ coincide with the analogous components of the tensor $g$ for the isotropic medium (Ilyushin, 1963). The function $\phi_{i j k l}$ characterizes the degree of plastic anisotropy. 
In the case of isotropic stress, $\sigma_{11}^{0}=\sigma_{22}^{0}=\sigma_{33}^{0}$, the coefficient $k$ becomes $k=0$ and $g_{i j k l}=g_{i j k l}^{0}$. For one-and two-axial stress we get five independent components of tensor $g_{i j k l}$.

$$
\begin{aligned}
& g_{11}=g_{11}^{0}\left(1+\frac{2}{7} k^{2} \frac{4 \lambda_{c}+11 \mu_{c}}{2 \lambda_{c}+7 \mu_{c}}\right) \\
& g_{33}=g_{33}^{0}\left(1+\frac{2}{7} k^{2} \frac{6 \lambda_{c}-\mu_{c}}{2 \lambda_{c}+7 \mu_{c}}\right) \\
& g_{12}=g_{12}^{0}\left(1+\frac{2}{7} k^{2}\right) \\
& g_{13}=g_{13}^{0}\left(1+\frac{6}{7} k^{2}\right) \\
& g_{23}=g_{23}^{0}\left(1-\frac{3}{7} k^{2} \frac{3 \lambda_{c}-2 \mu_{c}}{3 \lambda_{c}+8 \mu_{c}}\right)
\end{aligned}
$$

where $\lambda_{c}$ and $\mu_{c}$ are the Lame parameters of the reference body.

In the above formulas, tensor indices are replaced by matrix indices.

According to formula (14), there are also five independent components of tensor $b^{c}$ :

$$
b^{c}=-c^{c}-g^{-1}
$$

where $c^{c}$ is the tensor of the elastic constant of the reference body.

From relations (14), we obtain five independent components of the tensor $c^{*}$ which

\begin{tabular}{|c|c|c|c|c|c|c|c|}
\hline$\eta$ & $\chi$ & $\rho^{2}$ & $c_{11}^{*}$ & $c_{12}^{*}$ & $c_{13}^{*}$ & $c_{33}^{*}$ & $C_{44}^{*}$ \\
\hline \multicolumn{8}{|c|}{$\mathrm{v}_{1}=0.1 / \mathrm{v}_{2}=0.9$} \\
\hline 1.0 & 0.5 & 1.00 & 0.800 & 0.298 & 0.298 & 0.800 & 0.293 \\
\hline \multirow[t]{3}{*}{1.5} & 0.5 & 0.44 & 0.681 & 0.240 & 0.284 & 0.759 & 0.293 \\
\hline & 0.9 & 0.73 & 0.892 & 0.349 & 0.363 & 0.916 & 0.315 \\
\hline & 1.0 & 0.81 & 0.937 & 0.372 & 0.381 & 0.952 & 0.321 \\
\hline \multirow[t]{2}{*}{2.0} & 0.9 & 0.60 & 0.873 & 0.341 & 0.361 & 0.910 & 0.315 \\
\hline & 1.0 & 0.72 & 0.925 & 0.366 & 0.380 & 0.948 & 0.321 \\
\hline \multirow[t]{3}{*}{3.0} & 0.9 & 0.39 & 0.840 & 0.327 & 0.358 & 0.899 & 0.315 \\
\hline & 1.0 & 0.63 & 0.913 & 0.361 & 0.378 & 0.943 & 0.321 \\
\hline & \multicolumn{7}{|c|}{$\mathrm{v}_{1}=0.5 / \mathrm{v}_{2}=0.5$} \\
\hline 1.0 & 0.5 & 1.00 & 1.455 & 0.557 & 0.557 & 1.455 & 0.515 \\
\hline \multirow[t]{4}{*}{1.5} & 0.5 & 0.41 & 1.266 & 0.469 & 0.535 & 1.389 & 0.515 \\
\hline & 0.9 & 0.48 & 1.561 & 0.627 & 0.660 & 1.628 & 0.548 \\
\hline & 1.0 & 0.51 & 1.625 & 0.659 & 0.687 & 1.681 & 0.556 \\
\hline & 1.5 & 0.90 & 1.914 & 0.797 & 0.801 & 1.920 & 0.594 \\
\hline 2.0 & 1.5 & 0.85 & 1.908 & 0.795 & 0.800 & 1.918 & 0.594 \\
\hline 3.0 & 1.5 & 0.82 & 1.905 & 0.795 & 0.800 & 1.917 & 0.594 \\
\hline
\end{tabular}
depend on the microelasticity zone concentrations, stress type, and the plastic deformation extents. To calculate $c^{*}$ by formula (13), modules of the third component are found by formula (12). Table 2 gives the components of the tensor of effective

Table 2 Components of the tensor of effective elasto-plastic stiffnesses calculated for two inclusion concentrations $v_{1}=0.1$ and $v_{1}=0.5$. 
Table 3 Elastic properties of an isotropic composite (Arkhipov et al. 1975).

\begin{tabular}{ccc}
\hline & $\mathrm{E}_{*}, 10^{5} \mathrm{MPa}, \mathrm{G}_{*}, 10^{5} \mathrm{MPa}$ \\
$\mathrm{V}_{1}$ & $\mathrm{E}_{*}$ & $\mathrm{G*}$ \\
\hline 0.1 & 1.27 & 0.480 \\
0.2 & 1.34 & 0.515 \\
0.5 & 1.53 & 0.588 \\
\hline
\end{tabular}

elastoplastic stiffnesses calculated for two inclusion concentration values $v_{1}=0.1$ and $v_{1}=0.5$. The values of $c_{i j}^{*}$ for $v_{1}$ values in the interval from 0.2 to 0.4 are omitted in the Table 2 , because the modulii are close to those for $v_{1}=0.1$.

Comparing the calculated data with the previously obtained experimental results (see Table 3) for a similar isotropic composite (Arkhipov et al., 1975) allows that for high concentrations of rigid inclusions the calculated and experimental results are in good agreement. The model itself gives the possibility for qualitative predicting the type and degree of induced anisotropy of the effective elastic properties in dependence on the type and amount of external stress.

\section{Acknowledgments}

The authors are grateful to Dr. K. Walther for valuable discussions and advise.

\section{References}

Allen A. T., Hutching M. T. and Windsor C. G. (1985). Neutron diffraction for the study of residual stress fields. Advances in Physics, 34, 445-473.

Arkhipov I. K. and Golovin S. A. (1975) Elastic and inelastic properties of powder composites and methods of their description. In: Golovin S.A. (Ed.), Problems of the Materials Science and Physics of Metals. Tula, 101-110.

Fink K. and Rorbach Kh. (1961). Measurement of stress and deformation. Moscow, Mashgiz, 534 p. Ilyushin A. A. (1963). Plasticity. Moscow, USSR ASc. Publishing House, 286 p.

Jonas J. J., L. S. Toth and T. Urabe. (1994). Modeling the static and dynamic recrystallization on texture development. Materials Science Forum. ICOTOM - 10, 157-162, part 2, 1713-1730.

Kasatkin B. S. et al. (1981). Experimental methods for investigation deformation and stress. Reference book, Kiev, Naukova Dumka, $581 \mathrm{p}$.

Nikitin A. N. and Arkhipov I. K. (1992). Modeling texture formation in quartz-containing rocks at a phase transition temperature. Izv. Akad. Nauk SSSR, Fizika Zemli, 12, 29-40.

Nikitin A. N. and Ivankina T. I. (1995). On the possible mechanisms of the formation of piezoelectric active rocks with crystallographic textures. Textures and Microstructures. (in press).

Siemes H. and Hennig-Michaeli Ch. (1985). Ore Minerals. In H. -R. Wenk (Ed.). Preferred orientation in deformed metals and rocks: An introduction to modern texture analysis. Ac. Press, Orlando, Florida, 335-360.

Shermergor T. D. (1977). The theory of elasticity of microinhomogeneous media. Moscow, Nauka, 399 p.

Webster G. A., Smith D. J., Legatt R. H. and Macgillivray H. J. (1988). Neutron diffraction measurements of residual stress and plastic deformation in an aluminium alloy weld, J. Strain Analysis, 201-211.

Wenk H. -R. (1985) Carbonates. In H. -R. Wenk (Ed.). Preferred orientation in deformed metals and rocks: An introduction to modern texture analysis. Ac. Press, Orlando, Florida, 361-384

Wenk H. -R. (1994) Development of deformation textures in rocks. Physics of the Solid Earth, published by $A G U$ and GSA, 29, 6, 461-489. 